\title{
Characterization of the cytotoxicity and imaging properties of second- harmonic nanoparticles
}

\author{
Chia-Lung Hsieh*a,b, Rachel Grange ${ }^{\mathrm{a}}$, Ye Pu ${ }^{\mathrm{a}}$, Demetri Psaltis ${ }^{\mathrm{a}}$ \\ ${ }^{a}$ School of Engineering, EPFL, Station 17, 1015 Lausanne, Switzerland \\ ${ }^{\mathrm{b}}$ Department of Electrical Engineering, California Institute of Technology, Pasadena, CA 91125, \\ USA
}

\begin{abstract}
We develop second-harmonic nanoparticles as the contrast agents for cell imaging. Second-harmonic nanoparticles show promise as cell imaging probes due to their non-bleaching, non-blinking, and coherent signal. Nanoparticles of noncentrosymmetric crystal structures have high second-harmonic generation (SHG) efficiency and provide high contrast in a generally non-structured cell environment. Here, we use barium titanate $\left(\mathrm{BaTiO}_{3}\right)$ nanoparticles with tetragonal crystal structure as imaging probes. Cytotoxicity tests performed on $\mathrm{BaTiO}_{3}$ nanoparticles with mammalian cells did not result in toxic effects. Specifically, we observed no change in the cell metabolism after 24 hours incubation of the cells with high concentration of $\mathrm{BaTiO}_{3}$ nanoparticles. We demonstrate two methods of cell labeling with $\mathrm{BaTiO}_{3}$ nanoparticles for imaging. One is non-specific labeling via endocytosis of the cells, which results in a great number of the nanoparticles randomly distributed inside the cells. The other is specific labeling via surface functionalization of the nanoparticles with antibodies, which enables us to label specific cell membrane proteins with the nanoparticles. SHG imaging is compatible to two-photon microscopy and the SHG signal from nanoparticles can be easily detected with a standard two-photon confocal microscope. Our work provides the opportunity for long-term, three-dimensional cell tracking with secondharmonic nanoparticles.
\end{abstract}

Keywords: Second harmonic generation, barium titanate, nanoparticle, microscopy, biological cells, cytotoxicity, endocytosis, surface functionalization

\section{INTRODUCTION}

Detecting and tracing specific molecules of interest are important for studying the function and the behavior of biological systems. Biomarkers have been developed to achieve this goal by creating a contrast between signal and background. An extremely effective method to obtain such contrast is to shift the radiated signal away from the excitation wavelength so that the excitation can be efficiently removed by optical filters. We have developed $\mathrm{BaTiO}_{3}$ nanoparticles as biomarkers using the SHG signal from nanoparticles. We refer to these probes as "Second Harmonic Radiation IMaging Probes (SHRIMPs)". When a nanoparticle of non-centrosymmetric crystal structure is optically excited at a fundamental frequency, it emits the optical signal at the exact doubled frequency. Only materials with crystalline structure lacking a center of symmetry are capable of efficient SHG, while centrosymmetric and isotropic materials have a weak SHG response mostly from the surface where the symmetry is broken ${ }^{1}$. Thus, when imaged at the second harmonic frequency, SHRIMPs provide an effective contrast mechanism between the markers and the generally unstructured or isotropic biological environment. Ordered and highly polarizable biological non-centrosymmetric structures, such as collagen fibers, have endogenous $\mathrm{SHG}^{2}$. However, in most biological cell components, the endogenous SHG from the cell interface layers is weak ${ }^{3}$.

Unlike fluorescence, the SHG process only involves virtual electron energy transition without nonradiative energy loss. Owing to this virtual transition process, SHRIMPs do not bleach over time and emit a stable, non-blinking signal. Furthermore, the response time of SHG process is in the femtosecond scale, which allows for the observation of fast dynamic processes over a long time. In addition, SHG is generally a non-resonant process which offers broad flexibility in the choice of excitation wavelength. The coherent nature of the SHG signal is also a major advantage, providing a possibility to detect the SHG signal from the nanoparticles with interferometric optical techniques ${ }^{4,5}$.

*chia-lung.hsieh@epfl.ch; phone 4121 693-7828.

Biosensing III, edited by Hooman Mohseni, Manijeh Razeghi, Proc. of SPIE Vol. 7759,

77590 T · 2010 SPIE · CCC code: 0277-786X/10/\$18 · doi: 10.1117/12.860595 
With all the advantages of SHRIMPs as biomarkers, it is critical to test the possible toxicity for the applications of the $\mathrm{BaTiO}_{3}$ nanoparticles in biological cells and tissues. In this paper, we report the cytotoxicity test on $\mathrm{BaTiO}_{3}$ nanoparticles in mammalian cells. In the test, we observe no change in the cell metabolism after 24 hours incubation of the cells with high concentration of $\mathrm{BaTiO}_{3}$ nanoparticles.

In cell imaging applications, non-specific labeling can be easily done via endocytosis of the cells by incubating the cells with high concentration of the nanoparticles. The excess nanoparticles in the medium can be separated from the labeled cells by centrifugation after the incubation. Here we demonstrate the non-specific labeling method with myeloid cells. A great number of SHRIMPs are observed randomly distributed inside the cells. For most biological applications, it is also necessary to prepare a chemical interface for specific labeling. The surface functionalization of the SHG active dielectric nano-materials can be limited by lack of compatibility between the inorganic nanoparticles and the organic biomolecules. We demonstrate the specific labeling of $\mathrm{BaTiO}_{3}$ nanoparticles by directly conjugating immunoglobulin $\mathrm{G}$ (IgG) antibody on the surface of the nanoparticles. The antibody conjugation also provides a flexible scheme to target a specific molecule in a biological specimen.

\section{CYTOTOXICITY TEST OF THE NANOPARTICLES}

We started with $\mathrm{BaTiO}_{3}$ nanoparticles in dry powder form. X-ray diffraction data (not shown here) confirms that the crystal structure is tetragonal, which is non-centrosymmetric, allowing for efficient SHG without any further treatment. A scanning electron microscope (SEM) image of $\mathrm{BaTiO}_{3}$ nanoparticles is shown in Figure 1. The nanoparticles are nearly spherical in shape and between $60 \mathrm{~nm}$ to $110 \mathrm{~nm}$ in diameter. The $\mathrm{BaTiO}_{3}$ nanoparticles were dispersed in water and treated with ultrasound (Branson digital sonifier 450) to break them into individual nanoparticles. Dynamic light scattering measurement (data not shown) indicates that the average size of the nanoparticles was $90 \mathrm{~nm}$ in diameter.

To improve the stability of the colloidal suspension, we functionalize the $\mathrm{BaTiO}_{3}$ nanoparticles by attaching primary amine groups on the surface of the nanoparticle. The primary amine group attached to the surface of an inorganic material is also a general cross-link to bio-molecules. The bare $\mathrm{BaTiO}_{3}$ nanoparticles were placed in $1 \mathrm{M}$ of nitric acid for 10 minutes to remove the surface barium ions. After the treatment, we have a " $\mathrm{TiO}_{2}$-like" particle surface. We then placed the treated particles in $10 \mu \mathrm{M}$ of 3-aminopropyltriethoxysilane (APTES) in ethanol-water-ammonia solution $75 / 23.5 / 1.5 \mathrm{v} / \mathrm{v} / \mathrm{v} \%$ at $70{ }^{\circ} \mathrm{C}$ for 8 hours. The organofunctional silanes act as a bridge between organic and inorganic phases by forming a covalent attachment of organic monolayers. As a result, we have accessible primary amine groups immobilized on the surface of $\mathrm{BaTiO}_{3}$ nanoparticles, referring to as amino- $\mathrm{BaTiO}_{3}$ nanoparticles.

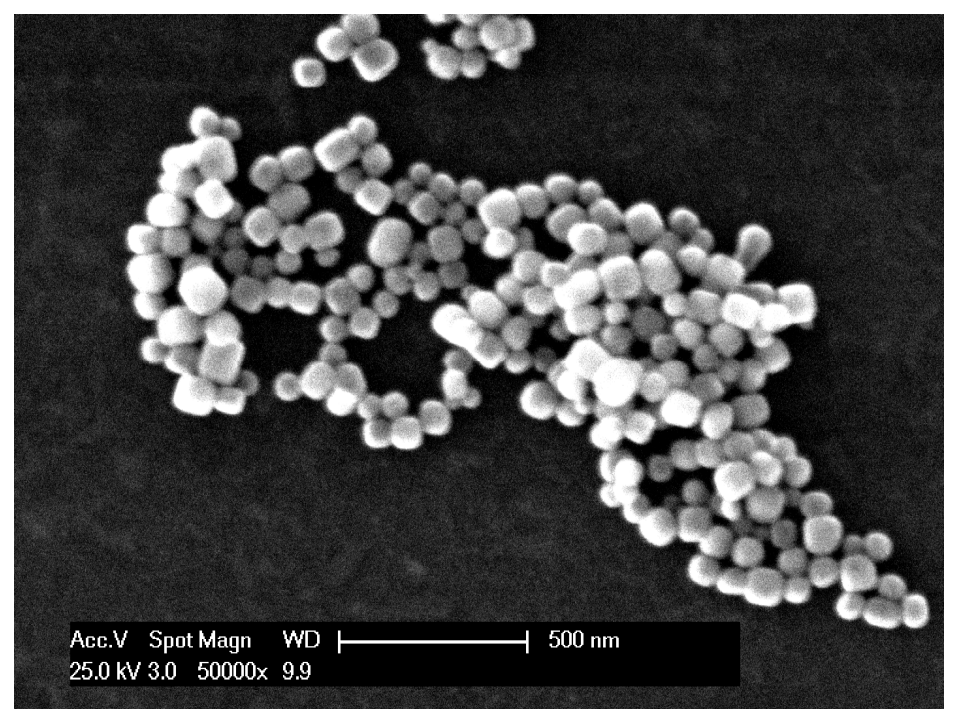

Figure 1. SEM image of the $\mathrm{BaTiO}_{3}$ nanoparticles. 
We test the cytotoxicity of the amino- $\mathrm{BaTiO}_{3}$ nanoparticles by measuring the cell viability after being incubated with the nanoparticles. HeLa cells were cultured in the growth media at the concentration of $\sim 6000 \mathrm{cells} / \mathrm{cm}^{2}$ in 96 -well polystyrene cell culture microplates (Greiner bio-one) for the test. Three different concentrations of the nanoparticles were added to the cell medium, which results in the overall concentrations of $10^{8}, 10^{9}$, and $10^{10}$ nanoparticles $/ \mathrm{cm}^{3}$ in the cell medium. The cells were incubated with the nanoparticles at $37^{\circ} \mathrm{C}$ in a humidified atmosphere of $5 \% \mathrm{CO}_{2}$ for 24 hours. After the incubation, we added 10\% of commercial available Alamar Blue (AbD Serotec) into the cell medium and incubated for 4 hours. Alamar Blue is popular as a simple and versatile indicator for measuring cell proliferation and cytotoxicity ${ }^{6}$. The oxidized, blue, nonfluorescent Alamar Blue is reduced to a pink fluorescent dye in the medium by cell activity (likely to be by oxygen consumption through metabolism). After the incubation, the fluorescent intensity of the cell medium, which reflects the cell viability, was measured by a monochromator microplate reader (Tecan, Safire2). The result is shown in Figure 2. We observed no change in the cell viability in three samples with different concentration of the nanoparticles, which indicates the $\mathrm{BaTiO}_{3}$ nanoparticles are non-toxic to the cells.

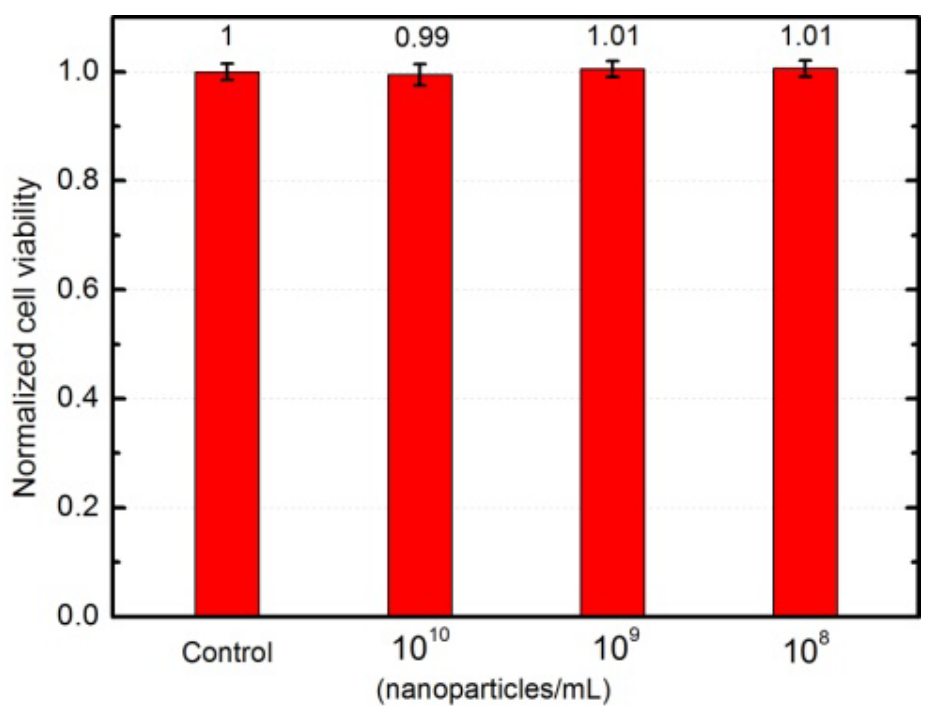

Figure 2. Cell viability measurement after incubating the $\mathrm{HeLa}$ cells with different concentrations of the $\mathrm{BaTiO}_{3}$ nanoparticles for 24 hours. The results have been normalized to the control experiment.

\section{NON-SPECIFIC CELL LABELING}

We demonstrate non-specific labeling of the cells with SHRIMPs via endocytosis of the cells. Endocytosis is the process by which cells uptake objects from outside the cells by engulfing them with their cell membrane. We incubated the SHRIMPs with CD11b+ myeloid cells for 12 hours for the endocytosis process. After that, the excess SHRIMPs were removed from the labeled cells by centrifugation. The cells were kept alive for another 16 hours before the imaging. A standard two-photon confocal microscope (Leica, SP5) was used to image the cells labeled by SHRIMPs. The excitation light source consisted of linearly polarized femtosecond laser pulses (Coherent, Chameleon) and the excitation wavelength was centered at $812 \mathrm{~nm}$ wavelength. The laser was focused by a 20x $1.0 \mathrm{NA}$ water-immersion microscope objective. The SHG signal was collected in epi-geometry in SHG channel (400-415 nm), and the transmission signal at the fundamental wavelength is also detected as the second channel. The results are shown in Figure 3 . The contrast of the cells in the transmission channel is low (Figure 3(a)) because the cells are almost transparent. In the SHG channel, we observed a large number of SHRIMPs randomly distributed inside the cells with high contrast (Figure 3(b)). 

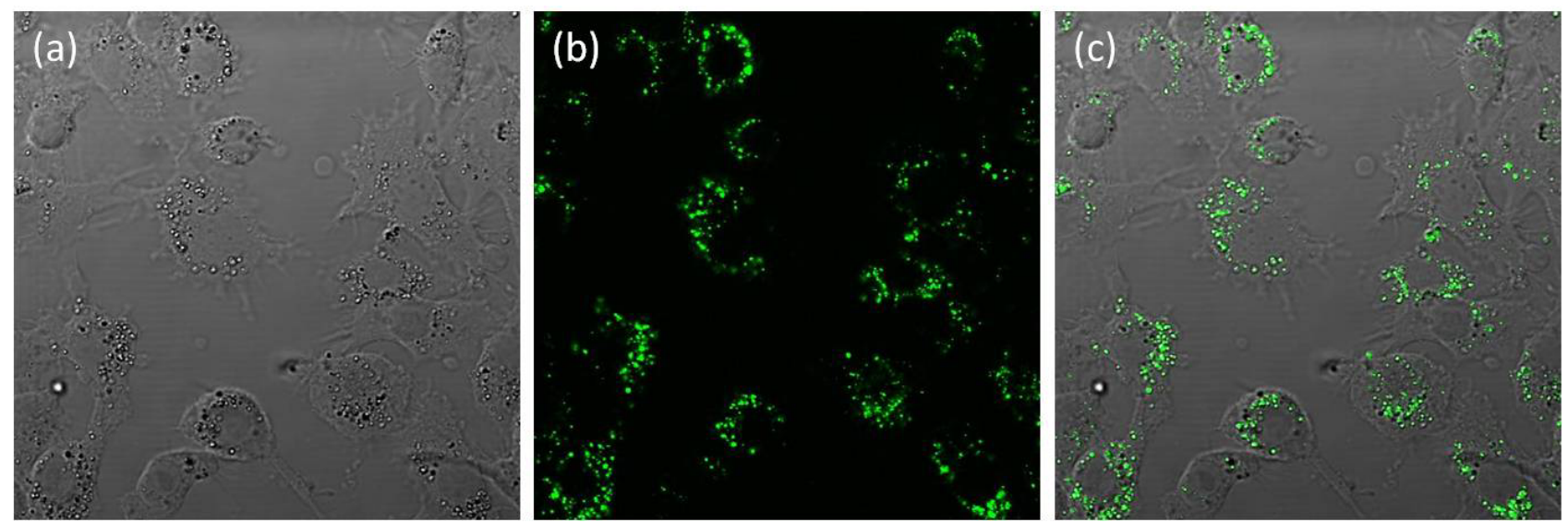

Figure 3. Scanning confocal section images of the SHRIMPs inside the myeloid cells via endocytosis. (a) Transmission channel (b) SHG channel (c) Merge image of (a) and (b). The size of the images is $130 \times 130 \mu^{2}$.

\section{SPECIFIC CELL MEMBRANE PROTEIN LABELING}

We demonstrate the specific labeling of SHRIMPs through an indirect staining process: the target molecule is first labeled with a primary antibody which is then recognized by a secondary-antibody-SHRIMP conjugate (2nd-AbSHRIMP). As described in Section 2, the primary amine group on the surface of the amino- $\mathrm{BaTiO}_{3}$ nanoparticles can serve as a general cross-link to organic molecules. The conjugation of the secondary IgG antibodies onto the amino$\mathrm{BaTiO}_{3}$ nanoparticles is based on the crosslinking reactions between amine and sulfhydryl groups catalyzed by maleimide, which has been reported previously for the bioconjugation of quantum dots ${ }^{7}$. The scheme of the conjugation process is shown in Figure 4. After the conjugation, we have secondary antibody covalently conjugated on the surface of the SHRIMP, namely 2nd-Ab-SHRIMPs. Highly specific labeling of the 2nd-Ab-SHRIMPs on a microarray of the primary antibodies was observed ${ }^{8}$ (less than $5 \%$ nonspecific labeling, data not shown).
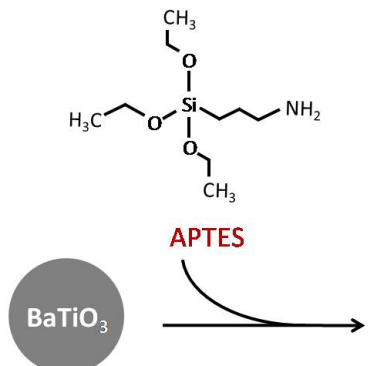

Amino- $\mathrm{BaTiO}_{3}$

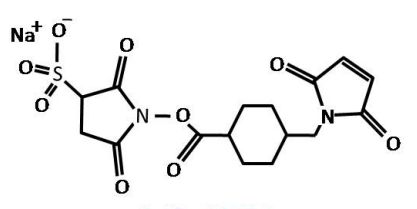

Sulfo-SMCC

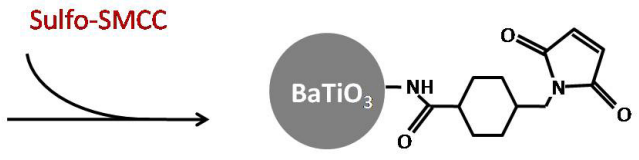

Maleimide-activated $\mathrm{BaTiO}_{3}$

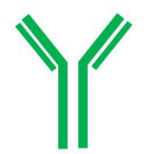

IgG antibody

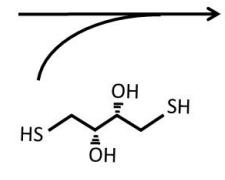

DTT
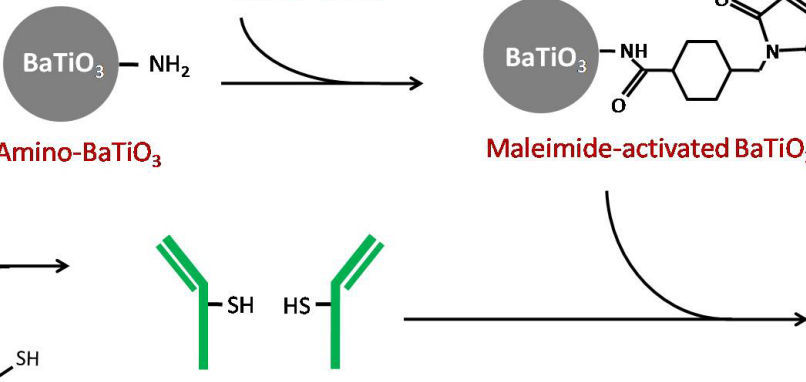

Reduced IgG antibody

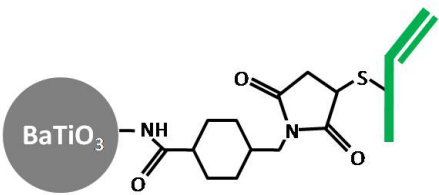

Antibody-SHRIMP conjugate

Figure 4. Scheme of covalent bioconjugation of $\mathrm{IgG}$ antibody onto the surface of $\mathrm{BaTiO}_{3}$ nanoparticle for specific labeling imaging application.

We examined the specific labeling of SHRIMPs in a biological cell environment. The human leukocyte antigen (HLA) class I molecule is critical to immune system function in humans because it represents antigenic peptides to T cells. We demonstrated the specific targeting of the HLA class I molecule presented on the HeLa cell surface with $\mathrm{BaTiO}_{3}$ nanoparticles. The HeLa cells were immunostained in suspension with the primary antibody specific to human HLA 
class I antigens followed by the staining of 2nd-Ab-SHRIMPs. For comparison, we prepared a control sample where HeLa cells were not stained by the primary antibody under otherwise identical conditions. The cells were prepared in a petri dish and kept alive during the imaging. The cells were imaged with a scanning confocal microscope (Leica, SP5) as described in the previous section.

Figure 5 (a)-(c) show the scanning section images of the cells labeled with 2nd-Ab-SHRIMPs. From these images, we observe a great number of 2nd-Ab-SHRIMPs attached on the cell surface. The SHRIMPs have a high contrast in biological cell environment since the endogenous SHG from the cell components is weak. It is worth noting that SHRIMPs emit SHG signal equally in the forward and backward directions due to the scattering nature of the process, so the epi-geometry is efficient for signal collection. For comparison, Figure 5 (d)-(f) show the section images of the HeLa cells in the control sample where almost no 2nd-Ab-SHRIMP is observed on the cell surface. It indicates that the labeling of 2nd-Ab-SHRIMPs on the cell membrane in Figure 5 (a)-(c) is specific to the primary antibody.

In conclusion, we developed $\mathrm{BaTiO}_{3}$ nanoparticles as cell imaging probes by using the SHG signal. The SHG signal from the nanoparticles is sufficient for a standard two-photon scanning confocal microscope, and it has high contrast in a cell environment. We showed the $\mathrm{BaTiO}_{3}$ nanoparticles are non-toxic to HeLa cells by performing the cytotoxicity test. No change of cell metabolism was observed after incubating the cells with high concentration of the nanoparticles for 24 hours. We demonstrated non-specific labeling of the cells with the nanoparticles via endocytosis of the cells, and also specific labeling of the cells via bioconjugation of the antibodies on the surface of the nanoparticles. We showed that with these two approaches, SHRIMPs provide the opportunities for long-term and three-dimensional cell tracking.
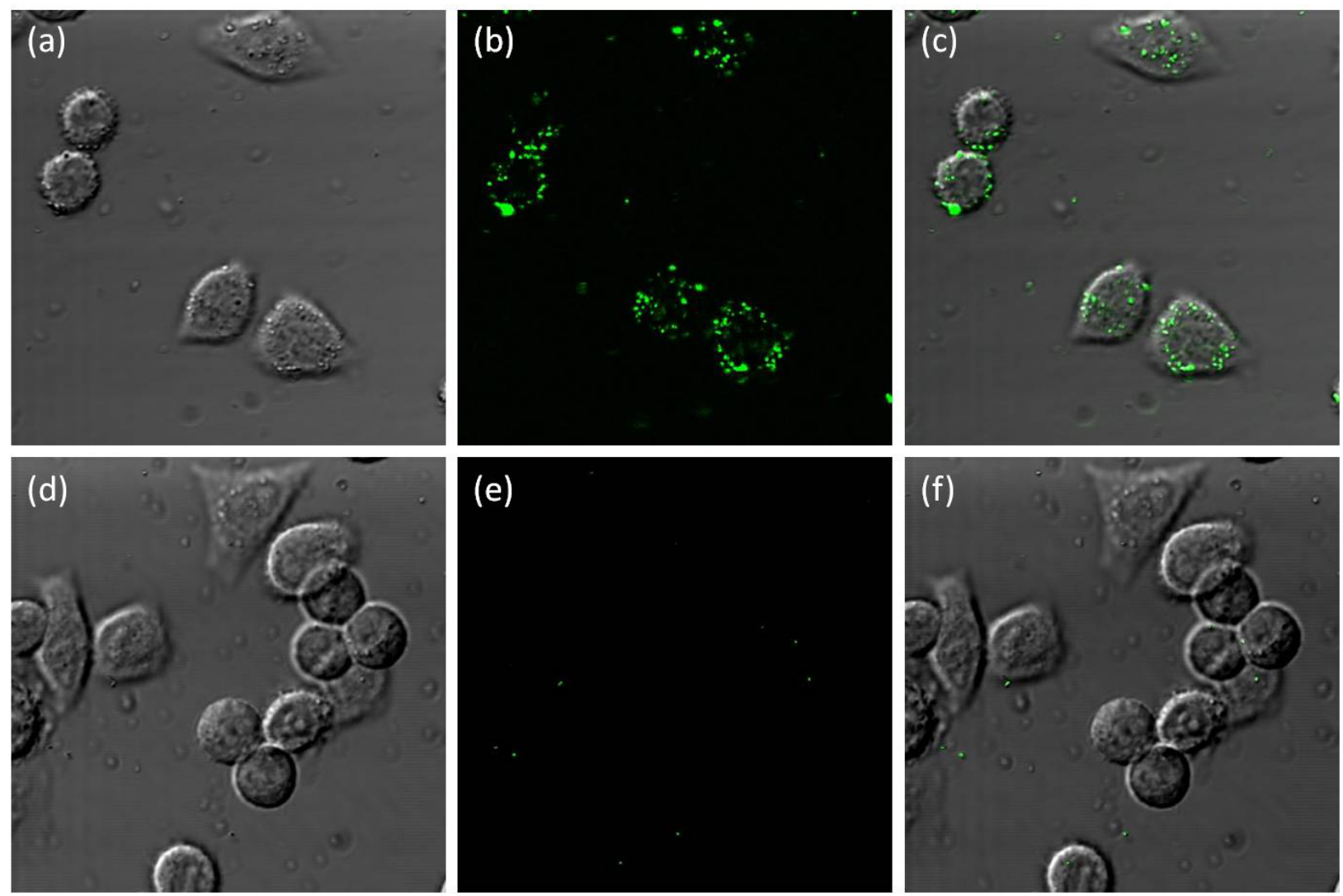

Figure 5. Scanning confocal section images of HeLa cells labeled with the 2nd-Ab-SHRIMPs. (a)-(c) The cells were first labeled with the primary antibody specific to the HLA class I molecules present on the cell membrane and then labeled with the 2nd-Ab-SHRIMPs; (d)-(f) A control sample where the cells were not labeled with primary antibody under otherwise identical conditions; transmission images are shown in (a) and (d); SHG images are shown in green in (b) and (e); and the merge images of transmission images and SHG images are shown in (c) and (f). The size of the images is $105 \times 105 \mu \mathrm{m}^{2}$. 


\section{ACKNOWLEDGEMENTS}

The authors thank Paul Bowen for providing the nanoparticles, Marc Chambon and Nathalie Ballanfat for the help of HeLa cell preparation and cytotoxicity test. We also like to thank Eric Bernasconi and Dominique Velin at the Gastroenterology division in CHUV for the myeloid cells preparation. This project is supported by National Center of Competence in Research (NCCR), Quantum Photonics.

\section{REFERENCES}

[1] Dadap, J. I., Shan, J., Eisenthal, K. B., Heinz, T. F., "Second-harmonic Rayleigh scattering from a sphere of centrosymmetric material," Phys Rev Lett 83(20), 4045-4048 (1999).

[2] Zipfel, W. R., Williams, R. M., Christie, R., Nikitin, A. Y., Hyman, B. T., Webb, W. W., "Live tissue intrinsic emission microscopy using multiphoton-excited native fluorescence and second harmonic generation," Proc Natl Acad Sci USA 100(12),7075-7080 (2003).

[3] Campagnola, P. J., Wei, M. D., Lewis, A., Loew, L. M., "High-resolution nonlinear optical imaging of live cells by second harmonic generation," Biophys J 77(6) 3341-3349 (1999).

[4] Pu, Y. Centurion, M., Psaltis, D., "Harmonic holography: a new holographic principle," Appl Optics 47(4) A103-A110 (2008).

[5] Hsieh, C.-L., Grange, R., Pu, Y., Psaltis, D., "Three-dimensional harmonic holographic microcopy using nanoparticles as probes for cell imaging," Opt Express 17(4) 2880-2891 (2009).

[6] PagêA, B., PageÂ, M., NoeÈl, C., "A new fluorimetric assay for cytotoxicity measurements in vitro," Int. J. Oncol. 3, 473-476 (1993).

[7] Xing, Y., Chaudry, Q., Shen, C., Kong, K. Y., Zhau, H. E., Wchung, L., et al., "Bioconjugated quantum dots for multiplexed and quantitative immunohistochemistry," Nat Protoc 2(5) 1152-1165 (2007).

[8] Hsieh, C.-L., Grange, R., Pu, Y., Psaltis, D., "Bioconjugation of barium titanate nanocrystals with immunoglobulin $\mathrm{G}$ antibody for second harmonic radiation imaging probes," Biomaterials 31(8), 2272-2277 (2010). 